\title{
Concomitant overexpression of EGFR and CXCR4 is associated with worse prognosis in a new molecular subtype of non-small cell lung cancer
}

\author{
ALYA A. AL ZOBAIR ${ }^{1,2,4}$, BARRAK F. AL OBEIDY ${ }^{2,4}$, LEI YANG ${ }^{2}$, CHUNXU YANG $^{2}$, \\ YANG HUI ${ }^{2}$, HAIJUN YU ${ }^{1,2}$, FANG ZHENG ${ }^{2}$, GUIFANG YANG ${ }^{3}$, \\ CONGHUA XIE ${ }^{1,2}$, FUXIANG ZHOU ${ }^{1,2}$ and YUNFENG ZHOU ${ }^{1,2}$ \\ ${ }^{1}$ Department of Radiation and Medical Oncology, Zhongnan Hospital of Wuhan University; \\ ${ }^{2}$ Hubei Key Laboratory of Tumor Biological Behavior, Hubei Cancer Clinical Study Center; \\ ${ }^{3}$ Department of Pathology, Zhongnan Hospital of Wuhan University, Wuhan 430071, P.R. China; \\ ${ }^{4}$ Department of Radiation and Medical Oncology, College of Medicine, Mosul University, Mosul, Iraq
}

Received October 1, 2012; Accepted December 12, 2012

DOI: $10.3892 / o r .2013 .2254$

\begin{abstract}
Although the relationships between CXCR4 and EGFR expression and survival in non-small cell lung cancer (NSCLC) have been studied independently, dual CXCR4/ EGFR tumor status and its relationship with survival has not been previously investigated. In the present study, we examined the relationship between CXCR4 expression, EGFR expression and dual CXCR4/EGFR expression and survival in patients with NSCLC $(n=125)$ using immunohistochemical techniques. Overall survival was estimated using Kaplan-Meier and Cox proportional hazards models adjusting for patient age, tumor stage and type of treatments. Patients with CXCR4-positive tumors were significantly associated with distant metastasis and tended to have poorer prognosis compared to patients with CXCR4-negative tumors $(\mathrm{HR}=2.172,95 \% \mathrm{CI}=1.229-3.839)$. No significant association between EGFR expression and survival was found; however co-expression of CXCR4/EGFR was a significant prognostic factor of worse overall survival ( $\mathrm{HR}=2.741,95 \% \mathrm{CI}=1.330-5.741)$. Furthermore, we showed that EGF enhanced the expression of CXCR4 in NSCLC cells through the PI-3K pathway, and treatment of NSCLC cells with EGFR phosphorylation inhibitor, AG1478, resulted in downregulation of the expression of CXCR4. These results suggest an important interaction between CXCR4 and EGFR intracellular pathways that may activate signals of tumor progression and may provide a valid explanation for the poor overall survival rate of patients whose co-expression of CXCR4 and EGFR is detected in tissue sections. Based on EGFR and CXCR4 expression, new molecular subtypes of NSCLC established in the present study can be used for customiza-
\end{abstract}

Correspondence to: Professor Yunfeng Zhou, Department of Radiation and Medical Oncology, Zhongnan Hospital of Wuhan University, 169 Road, Hubei, Wuhan 430071, P.R. China

E-mail: yfzhouwhu@163.com

Key words: NSCLC, CXCR4, EGFR, CXCL12, transactivation tion of NSCLC treatment. Our results also showed that EGFR and CXCR4 are potential therapeutic targets for NSCLC and that simultaneous inhibition of EGFR and CXCR4 in NSCLC patients with concomitant expression of both CXCR4 and EGFR may be an effective treatment strategy.

\section{Introduction}

Lung cancer, and particularly non-small cell lung cancer (NSCLC), is the leading cause of cancer-related mortality worldwide. In China, approximately 500,000 patients were diagnosed with lung cancer and 430,000 deaths were reported in 2005. In 2025, the number of deaths is expected to be more than one million (1-4). After diagnosis, less than $15 \%$ of patients survive beyond 5 years. Therefore, identification of molecular pathways involved in lung tumorigenesis and metastasis may lead to new targeted therapies. Among the known pathways are CXCR4 and EGFR, where both proteins are expressed in NSCLC and may predict prognosis $(1,5)$.

Chemokines are small secreted chemotactic (8-10 kDa) proteins which play an important role in the regulation of leukocyte trafficking and extravasations toward the sites of tissue inflammation (6). Many recent reports have shown that these chemokines are involved in every aspect of cancer biology (7). Among chemokines and their receptors is the CXCL12/CXCR4 system. CXCR4 is a seven-transmembrane trimeric G-protein-coupled receptor (GPCR); CXCL12, also called stromal cell-derived factor $1 \alpha(\operatorname{SDF} 1-1 \alpha)$, is a powerful chemoattractant cytokine that directs locomotion of hematopoietic and non-hematopoitic cells $(8,9)$. Interaction between the chemokine receptor CXCR4 and its cognate ligand CXCL12 has been implicated in tumorigenicity, cell proliferation, angiogenesis and metastasis in 23 types of cancers, such as lung cancer, breast cancer, melanoma, glioblastoma, pancreatic cancer, colorectal carcinoma, cholangiocarcinoma basal cell carcinoma cells and prostate cancer, and it has been proposed as a prognostic marker for these malignancies (10-13). 
All NSCLC cell lines have been shown to express CXCR4 on the cell surface (14). Kijima et al reported that the expression of the CXCR4 receptor in NSCLC cells regulates their homing to organs that express higher levels of CXCL12 such as lung, liver, bone marrow and lymph node. Similarly, Phillips et al reported that the CXCR4/CXCL12 biological axis can regulate the overall metastatic behavior of NSCLC, and they found that in vivo neutralization of CXCL12 in an SCID mouse system of human NSCLC by anti-CXCL12 antibodies resulted in a marked attenuation of NSCLC metastasis to adrenal gland, lymph node, liver, lung and bone marrow $(15,16)$.

Moreover, NSCLC patients with clinical metastasis have been shown to express a high level of CXCR4 mRNA in their tissue specimen compared with patients without metastasis, suggesting that the activation of the CXCR4/CXCL12 pathway increases the ability of metastasis-associated behavior such as cell invasion and migration $(14,17,18)$. Oonakanava et al found that the CXCL12/CXCR4 axis is involved in the metastasis of NSCLC cells into the pleural space (14), while Tang et al reported that the CXCL12/CXCR4 pathway mediates bone-specific metastasis of NSCLC (8). Wagner et al reported that cytomembranous expression of CXCR4 is an independent risk factor associated with poor outcome in adenocarcinoma (ADC) of the lung (19).

Epidermal growth factor receptor (EGFR) is a membrane bound receptor, which is frequently mutated or functions anomalously in cancer. These receptors homodimerize or heterodimerize, after the binding of their cognate ligands, resulting in activation of their tyrosine kinase domain, subsequently initiating a cascade of signals that affects cell cycle progression, angiogenesis, apoptosis and metastasis leading to the development and progression of cancer $(20,21)$. NSCLC tissues have been shown to overexpress EGFR at all tumor stages compared with uninvolved lung tissues. The EGFR gene copy number and EGFR mRNA transcript level are closely associated with the expression of EGFR in NSCLC tumors $(5,22)$. The frequency of EGFR overexpression has been reported to range from 34 to $84 \%$, and contradictory results have been reported regarding the correlation between EGFR overexpression and clinicopathological factors or patient survival. Either shorter or longer survival associated with EGFR expression has been reported by several studies (23). Despite the implication of EGFR signaling in the progression of NSCLC, a marginal survival benefit and limited response rates have been demonstrated with the use of anti-EGFR targeted therapies (24-26).

Interaction has been found between CXCR4 and EGFR in cancer cells. In ovarian cancer,EGFR has been shown to enhance the expression of CXCR4 in ovarian cancer cell lines through the activation of $\operatorname{Src}$ kinase that enhances tumor growth (27). Another study showed that EGFR activates not only CXCR4 but also MMP9, leading to the increased metastatic potential of tumors (28). Similarly, concerning breast cancer, the activation of both EGFR and ErbB2 has been shown to increase CXCR4 expression in breast cancer cells (29). Furthermore, it was found that patients with tumors co-expressing CXCR4 and EGFR had a high incidence of inflammatory breast cancer-related death and a lower overall survival rate (30). Regarding colon cancer, co-expression of both EGFR and CXCR4 has been shown to be positively correlated with lymph node metastasis and distant metastasis, when compared with high expression of each molecule alone (31). However dual expression of EGFR and CXCR4 and its relationship with prognosis has not been previously investigated in NSCLC.

The mechanism of EGFR-induced CXCR4 activation appears to be complex, yet a close relationship has been suggested. Therefore, we carried out the present study using NSCLC tissue samples and cell lines to evaluate the potential correlation between CXCR4 and EGFR, and to investigate the relationship between their expression and clinicopathological parameters and to evaluate the prognostic impact of individual and combined expression of CXCR4 and EGFR in NSCLC.

\section{Materials and methods}

Patients. We retrospectively selected 125 patients with a histological diagnosis of NSCLC at Zhong Nan Hospital who were followed-up on a regular basis during a qualified follow-up program lasting from 2003 to 2011. Tumors were staged according to the American Joint Committee on Cancer (AJCC) pathologic tumor-node-metastasis (TNM) classification. The study was conducted in conformity to the Helsinki Declaration and was approved by the Ethics Committee of Zhongnan Hospital, Wuhan, China.

Patient characteristics and recorded clinical features including age, gender, type of surgery, eventual concomitant treatment and evidence of recurrence were obtained from office files and hospital records. Follow-up was carried out on an outpatient basis at 3-month intervals for the first 2 years and thereafter, for 6-month intervals. The follow-up evaluation consisted of a physical examination and blood examination including the detection of pertinent tumor markers and contrast-enhanced computed tomography (CT) scan of the chest. Further evaluation including $\mathrm{CT}$ of the abdomen, bone scintigraphy and MRI of the brain were performed when any signs or symptoms of recurrence were observed. Recurrences were detected by imaging techniques and were confirmed histologically when necessary. Neither chemotherapy nor radiotherapy were administered prior to surgery, and none of the patients received EGFR-targeted anticancer therapy during the follow-up period.

Tissue preparation and immunohistochemical analysis. Immunohistochemical staining by a standard streptavidin-peroxidase technique was used to examine CXCR4 and EGFR expression. Briefly, 5- $\mu \mathrm{m}$ paraffin sections were made, deparaffinized in xylene and dipped in a graded series of ethanol. In order to block endogenous peroxidase activity, the slides were incubated in $3 \% \mathrm{H}_{2} \mathrm{O}_{2}$ for $10 \mathrm{~min}$, for antigen retrieval; deparaffinized sections were boiled in citric acid, $\mathrm{pH} 6.0$, in a pressure cooker for $3 \mathrm{~min}$. The slides were dipped and cleansed in Tris buffer and incubated with anti-CXCR4 antibodies (Abcam, ab2074) or with antiEGFR antibodies (Abcam, ab2430) overnight at $4^{\circ} \mathrm{C}$. The bound antibody was detected with a biotinylated secondary antibody (Maixin-Bio). The enzyme complex was visualized with 3,3'-diaminobenzidine tetrachloride. Negative control experiments included omitting the primary antibody and replacing it with normal serum. The slides were assessed by two pathologists in a blinded manner. 


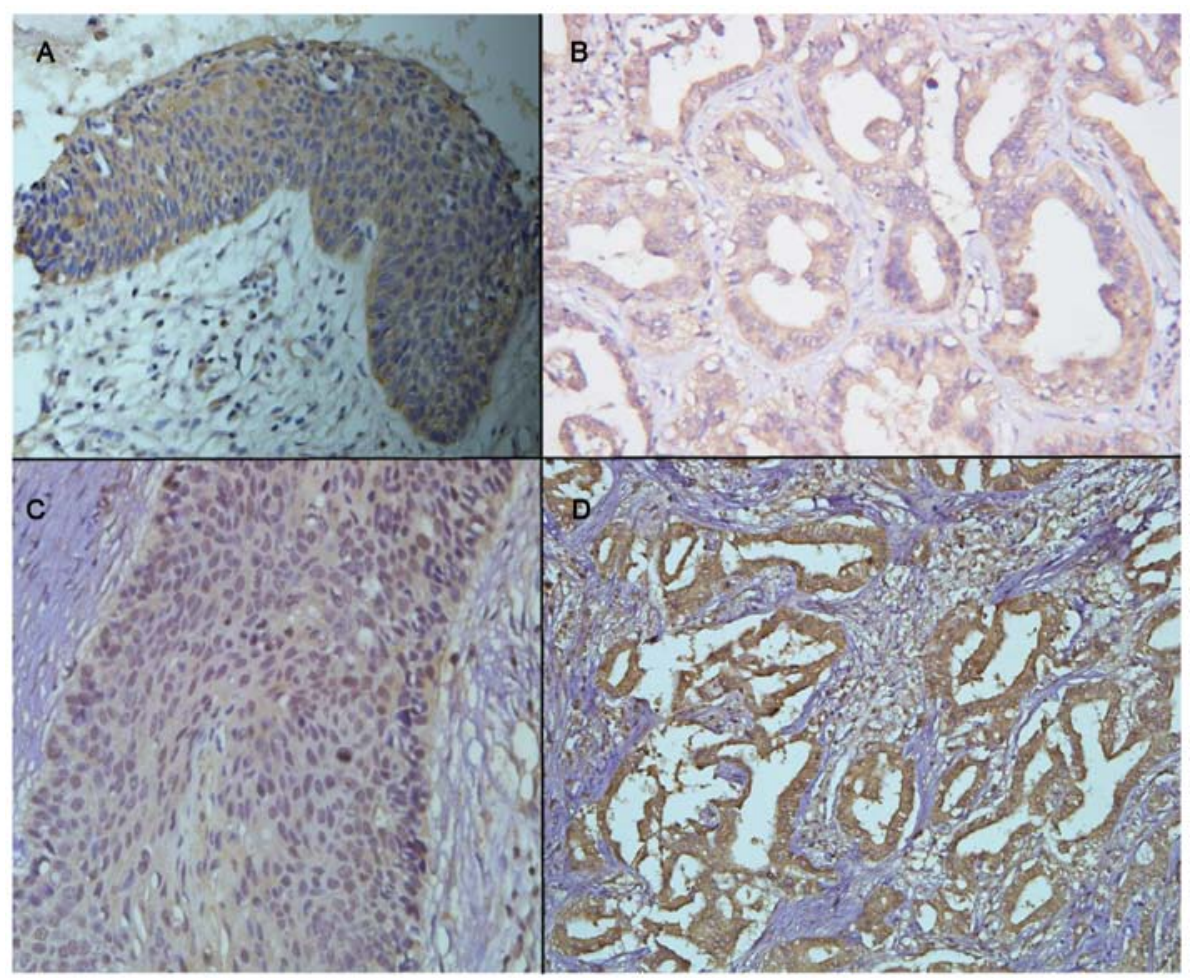

Figure 1. Immunohistochemical staining for EGFR and CXCR4. (A) Strong cytomembranous EGFR staining in squamous cell carcinoma (SCC) (x400). (B) Cytomembranous EGFR staining in adenocarcinoma (ADC) (x400). (C) Cytoplasmic CXCR4 staining in SCC (x400). (D) Strong cytoplasmic CXCR4 staining in $\mathrm{ADC}(\mathrm{x} 200)$.

Specific immunoreactivity was observed in the nuclei and cytoplasm of the tumor cells. We used a system that was based on staining intensity and the percentage of stained cells, as previously described for EGFR (33) and for CXCR4 (32). As shown in Fig. 1, IHC was regarded as positive only when evident distinct cell membrane staining was noted. An average of 1,500 cells per section was evaluated using a semi-quantitative grading system based on four scores ( 0 , no staining; $1+$, staining in $1-10 \%$ of the scrutinized cells; $2+$, staining in $11-25 \%$ of the scrutinized cells; $3+$, staining in $>25 \%$ of the scrutinized cells). In order to avoid inclusion of scattered positivity of the same intensity found in the normal bronchial tissue, a cutoff value of $10 \%$ positive cells was used.

Cell line. The human NSCLC cell line A549 was obtained from the Shanghai Institute of Health Sciences (Shanghai, China), The A549 cell line was cultured in RPMI-1640 media together with $1 \mathrm{mM}$ L-glutamine, $25 \mathrm{mM}$ HEPES buffer, $100 \mathrm{ng} / \mathrm{ml}$ streptomycin, $100 \mathrm{U} / \mathrm{ml}$ penicillin and $10 \%$ FCS (RPMI complete media).

Antibodies and reagents. The anti-CXCR4 and anti-EGFR antibodies were purchased from Abcam (Hong Kong). Recombinant human EGF was obtained from Peprotech (USA). LY294002 (PI-3K inhibitor) was from Beyotime Institute of Biotechnology (Jiangsu, China) and AG1478 from Sigma (St. Louis, MO, USA).

Real-time PCR. Total RNA was isolated from A549 cells using TRIzol reagent (Invitrogen) according to the manufacturer's instructions. Cells were lysed in TRIzol reagent and then mixed with chloroform, to separate the RNA, DNA and protein. The lysates were then centrifuged. Total RNA was precipitated with isopropanol, and then the RNA pellet was washed with $75 \%$ ethanol and subsequently dissolved in water. RNA $(1.5 \mu \mathrm{g})$ was reverse transcribed into cDNA using a Revert Aid cDNA synthesis kit, following the manufacturer's instructions. Finally, the cDNA was evaluated for changes in CXCR4 expression by real-time PCR using the Takara SYBR First Strand RT-PCR kit and MX3000P (Stratagene, La Jolla, CA, USA).

Western blotting. Using SDS-PAGE, immunoblotting was performed using $40 \mu \mathrm{g}$ of total protein. The proteins were transferred to PVDF membranes by electrophoresis at $100 \mathrm{~V}$ for $1 \mathrm{~h}$ at room temperature and subsequently blocked in non-fat milk for $30 \mathrm{~min}$. Following this, the membranes were incubated overnight at $4{ }^{\circ} \mathrm{C}$ with rabbit anti-human CXCR4 (ab2074; 1:1500; Abcam, Cambridge, CA, USA) and the blots were washed in TTBS before incubation with the secondary antibodies (goat anti-rabbit IgG-HRP) at a dilution of 1:3000 for $40 \mathrm{~min}$. After washing in TTBS, detection was carried out using enzyme linked chemiluminescence (ECL) detection reagents (Beyotime Institute of Biotechnology). To demonstrate equal loading of each lane, the membranes were then reprobed with a $\beta$-actin antibody (1:500; Santa Cruz Biotechnology, Inc., Santa Cruz, CA, USA).

Statistical analysis. The statistical analysis and data management were conducted using SPSS for Windows (version 18; SPSS). The $\chi^{2}$ test was used to assess significant clinicopathological differences between patients with positive and negative EGFR and CXCR4 expression. 
Table I. Correlation of EGFR and CXCR4 expression with clinicopathological features of the NSCLC cases.

\begin{tabular}{|c|c|c|c|c|c|c|c|c|c|c|}
\hline \multirow[b]{2}{*}{ Variables } & \multirow[b]{2}{*}{$\mathrm{N}$} & \multicolumn{3}{|c|}{ EGFR } & \multicolumn{3}{|c|}{ CXCR4 } & \multicolumn{3}{|c|}{ EGFR/CXCR4 } \\
\hline & & $+(\%)$ & $-(\%)$ & P-value & $+(\%)$ & $-(\%)$ & P-value & Both $+(\%)$ & Others & $\mathrm{P}$-value \\
\hline Total no. & 125 & 39.2 & 60.8 & & 49.6 & 50.4 & & 26.4 & 73.6 & \\
\hline \multicolumn{11}{|l|}{ Gender } \\
\hline Male & 87 & 30.4 & 39.2 & & 33.6 & 36.0 & & 20.8 & 48.8 & \\
\hline Female & 38 & 8.8 & 21.6 & 0.163 & 16.0 & 14.4 & 0.70 & 5.6 & 24.8 & 0.196 \\
\hline \multicolumn{11}{|c|}{ Histologic subtype } \\
\hline $\mathrm{ADC}$ & 64 & 16.0 & 35.2 & & 27.2 & 24 & & 14.4 & 36.8 & \\
\hline SCC & 61 & 23.2 & 25.6 & 0.098 & 22.4 & 26.4 & 0.419 & 12.0 & 36.8 & 0.654 \\
\hline \multicolumn{11}{|c|}{ Metastasis } \\
\hline Presence & 31 & 12.8 & 12.0 & & 20.8 & 4.0 & & 12.0 & 12.8 & \\
\hline Absence & 94 & 26.4 & 48.8 & 0.137 & 28.8 & 46.4 & 0.001 & 14.4 & 60.8 & 0.002 \\
\hline \multicolumn{11}{|c|}{ Tumor stage } \\
\hline I & 16 & 3.2 & 9.6 & & 2.4 & 10.4 & & 1.6 & 11.2 & \\
\hline II & 23 & 4.8 & 13.6 & & 5.6 & 12.8 & & 1.6 & 16.8 & \\
\hline III & 55 & 16.8 & 27.2 & & 20.8 & 23.2 & & 11.2 & 32.8 & \\
\hline IV & 31 & 14.4 & 10.4 & 0.053 & 20.8 & 4.0 & 0.001 & 12.0 & 12.8 & 0.005 \\
\hline
\end{tabular}

ADC, adenocarcinoma; SCC, squamous cell carcinoma.
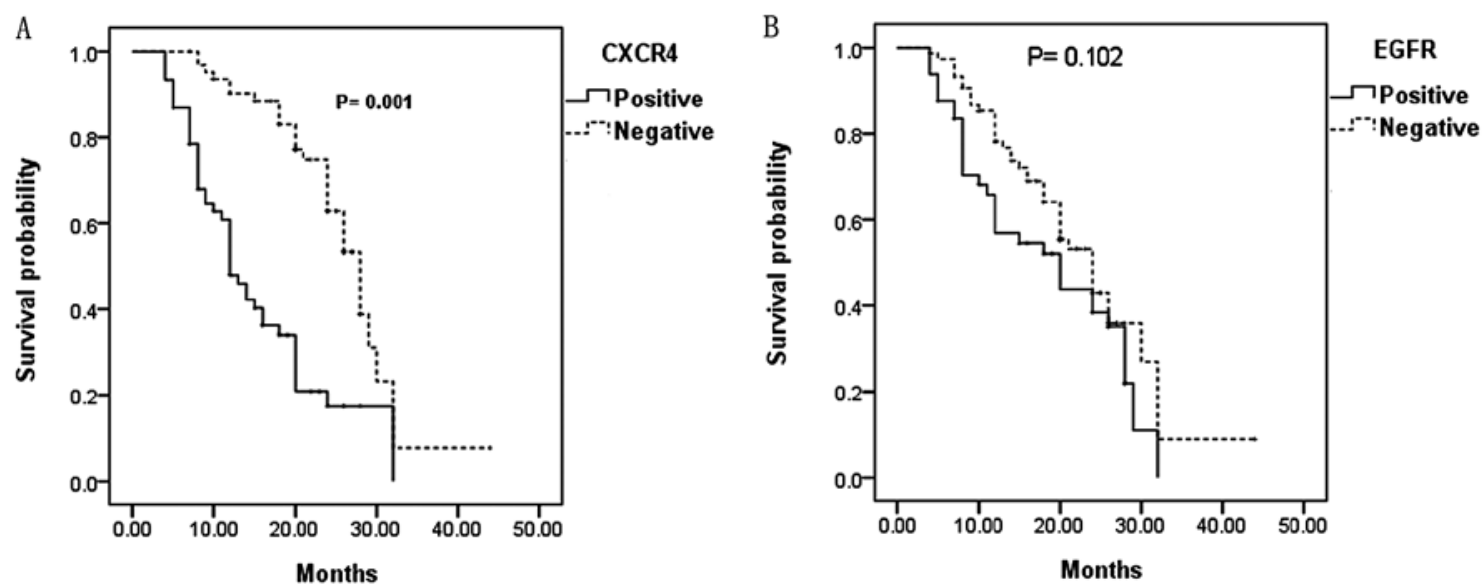

Figure 2. Kaplan-Meier estimates for overall survival according to (A) CXCR4 and (B) EGFR expression.

The duration of overall survival was calculated from the date of first diagnosis of the disease to the date of death or the last follow-up. Kaplan-Meier test was performed for survival analysis, and Cox proportional hazards models were used to evaluate the relationship between the tumor expression of CXCR4 and EGFR and the prognosis of NSCLC patients. Significant prognostic variables such as patient age, tumor stage and type of treatment were included in these models. Cox regression plots were constructed for $\mathrm{CXCR}^{+}{ }^{-}$vs. $\mathrm{CXCR}^{-}$and $\mathrm{EGFR}^{+}$vs. EGFR- The following additional comparisons were made: $\mathrm{CXCR}^{+} / \mathrm{EGFR}^{+}$vs. $\mathrm{CXCR}^{-} / \mathrm{EGFR}^{-}, \mathrm{CXCR}^{+} / \mathrm{EGFR}^{+}$ vs. others, $\mathrm{CXCR}^{+} / \mathrm{EGFR}^{-}$vs. others, $\mathrm{CXCR} 4^{-} / \mathrm{EGFR}^{+}$vs. others, CXCR4 ${ }^{-} / \mathrm{EGFR}^{-}$vs. others.

\section{Results}

The median age of the patients was 59 years (range 37-80), and the majority of the patients were male $(70 \%)(\mathrm{M}: \mathrm{F}=6: 1)$. Of the 125 examined biopsies, distribution of the histological types were 64 (51.2\%) ADCs and 61 (48.8\%) squamous cell carcinomas (SCCs). After diagnosis, 16 (12.8\%) patients were treated with surgery alone, 27 patients $(21.6 \%)$ were treated with surgery followed by radiochemotherapy, 48 patients $(38.4 \%)$ were treated with surgery followed by chemotherapy, 27 patients $(21.6 \%)$ were treated with chemoradiotherapy and 7 patients $(5.6 \%)$ were treated with chemotherapy. 
Table II. Estimated risk of death associated with EGFR and CXCR4 positivity in NSCLC.

\begin{tabular}{|c|c|c|c|}
\hline Strata & Hazard ratio & Confidence interval (95\%) & P-value \\
\hline CXCR4 & 2.172 & $1.229-3.839$ & 0.008 \\
\hline EGFR & 1.334 & $0.819-2.664$ & 0.248 \\
\hline $\mathrm{CXCR}^{+} / \mathrm{EGFR}^{+}$vs. others & 2.415 & $1.399-4.170$ & 0.01 \\
\hline $\mathrm{CXCR}^{+} / \mathrm{EGFR}^{-}$vs. others & 1.197 & $0.680-2.107$ & 0.533 \\
\hline CXCR4/EGFR ${ }^{+}$vs. others & 0.953 & $0.295-1.718$ & 0.199 \\
\hline CXCR4/EGFR ${ }^{-}$vs. others & 0.688 & $0.394-1.200$ & 0.188 \\
\hline $\mathrm{CXCR}^{+} / \mathrm{EGFR}^{+}$vs. CXCR4//EGFR & 2.741 & $1.330-5.741$ & 0.006 \\
\hline
\end{tabular}

Adjusted for patient age at diagnosis, tumor stage and type of treatments.
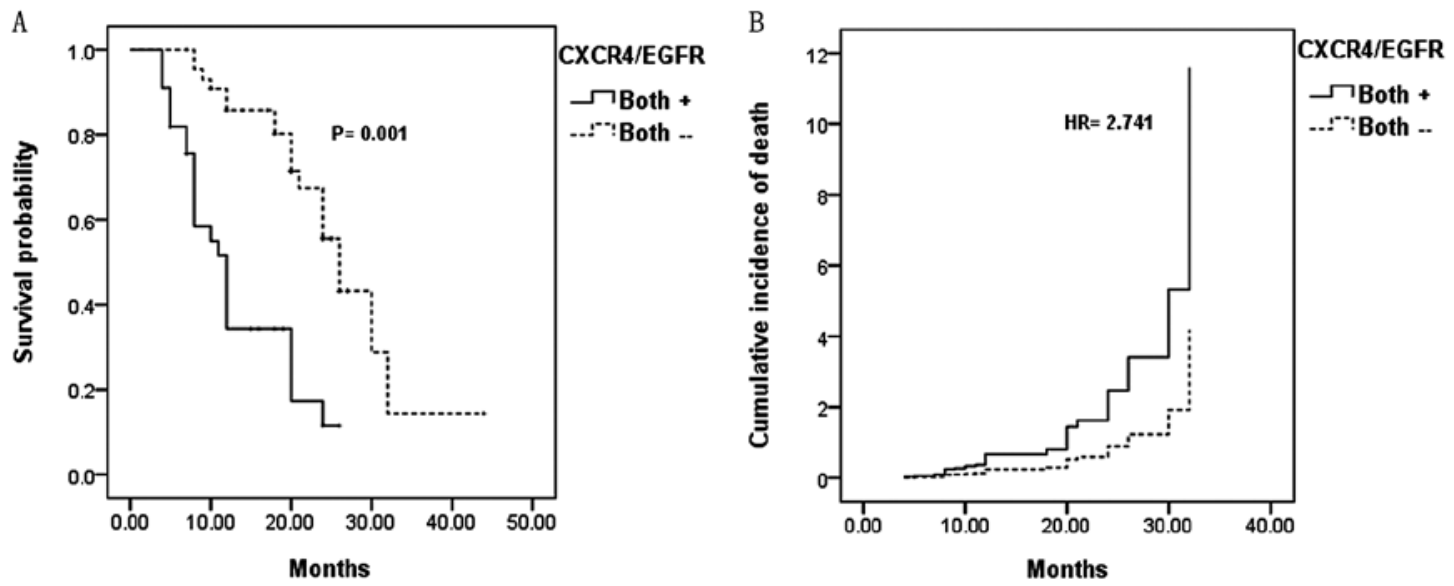

Figure 3. (A) Kaplan-Meier estimates for overall survival according to EGFR ${ }^{+}$CXCR4 $4^{+}$tumor expression. (B) Cox proportional hazards estimates for overall survival among patients with $\mathrm{EGFR}^{+} / \mathrm{CXCR}^{+}{ }^{+}$vs. EGFR ${ }^{-} / \mathrm{CXCR}^{-}$- tumors.

EGFR and CXCR4 protein expression in NSCLC samples. The positive frequency of EGFR and CXCR4 expression and co-expression of both receptors are shown in Table I. Using immunohistochemical techniques, EGFR expression was detected as a membranous and cytoplasmic staining pattern. Forty-nine (39.2\%) cases showed high expression and 76 $(60.8 \%)$ cases showed low expression. CXCR4 expression was detected as a cytoplasmic and nuclear staining pattern. Out of the 125 cases, 62 (49.6\%) showed high cytoplasmic expression while $63(50.4 \%)$ showed low cytoplasmic expression. High nuclear expression was found in 19 cases $(15.2 \%)$.

Dual EGFR/CXCR4 protein expression for the 125 cases of NSCLC included: CXCR4 ${ }^{+} / \mathrm{EGFR}^{+}$in 33 (26.4\%) cases, $\mathrm{CXCR}^{-} / \mathrm{EGFR}^{+}$in $16(12.8 \%)$ cases, $\mathrm{CXCR}^{+} / \mathrm{EGFR}^{-}$in $29(23.2 \%)$ cases and CXCR4/EGFR- in $47(37.6 \%)$ cases.

Clinicopathological characteristics. Neither EGFR tumor expression nor CXCR4 tumor expression were associated with the gender of the patients or histological subtype. Overexpression of the CXCR4 protein was observed more frequently in patients with clinical metastasis than in patients without metastasis $(\mathrm{P}=0.001)$, and CXCR4 was noted more frequently in advanced stage than in early stage NSCLC $(\mathrm{P}=0.001)$. EGFR overexpression was also found to be more frequent in advanced stage than in a localized stage, although this difference was not statistically significant $(\mathrm{P}=0.053)$. Thirty-three patients (26.4\%) showed concomitant overexpression of the EGFR and CXCR4 receptors. This group (CXCR4 ${ }^{+}$/ $\mathrm{EGFR}^{+}$) was also significantly associated with metastatic and advanced stage disease $(\mathrm{P}=0.002, \mathrm{P}=0.005$, respectively).

Survival analysis. The median survival time for cases with $\mathrm{CXCR}^{+}{ }^{+}$was 12.8 months and 21.1 months for patients with CXCR4-, while the median survival time for cases with nuclear staining of CXCR4 was 24 months and 16.4 months for patients with no nuclear staining of CXCR4. The median survival time for patients with $\mathrm{EGFR}^{+}$was 15.8 months and 16.2 months for patients with EGFR-. Patients with $\mathrm{CXCR}^{+}$ tumors had statistically significant shorter overall survival when compared with patients with $\mathrm{CXCR}^{-}{ }^{-}$tumors $(\mathrm{P}=0.001)$ (Fig. 2). Moreover, patients with $\mathrm{CXCR}^{+}$tumors had a statistically significant higher cumulative incidence of cancer-related death than patients with $\mathrm{CXCR} 4^{-}$tumors $(\mathrm{HR}=2.172)$, whereas there was no significant difference in overall survival between patients with positive or negative immunostaining for EGFR (Fig. 2).

The different association patterns for CXCR4 and EGFR protein expression were also compared. Patients were divided into 4 groups: group I with positive CXCR4 and positive EGFR $\left(\mathrm{CXCR}^{+} / \mathrm{EGFR}^{+}\right)$, group II with positive CXCR4 and negative 
A

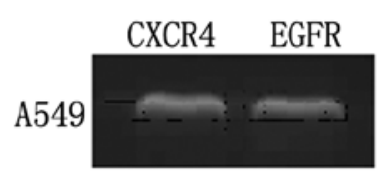

$\mathrm{C}$

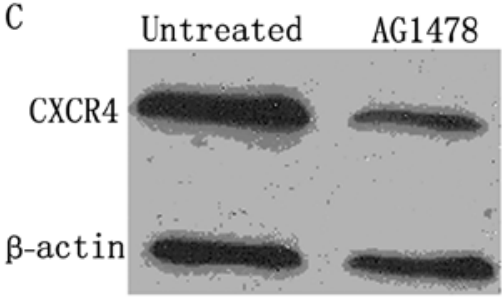

B

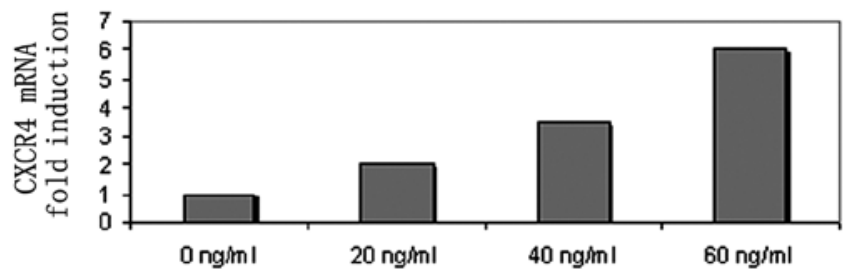

D

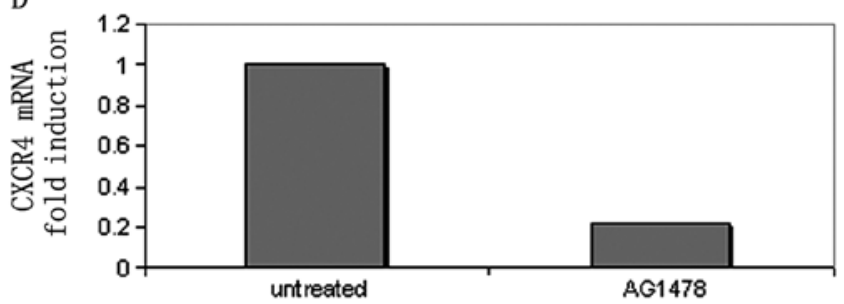

Figure 4. (A) RT-PCR analysis of mRNA expression of EGFR and CXCR4 in A549 cells. (B) CXCR4 expression after treatment for 24 h with various concentrations of EGF $(20,40$ and $60 \mathrm{ng} / \mathrm{ml})$ by real-time PCR. (C) CXCR4 expression after treatment with AG1478 (10 $\mu \mathrm{M})$ for $24 \mathrm{~h}$ as detected by western blotting. (D) CXCR4 expression after treatment with AG1478 $(10 \mu \mathrm{M})$ for $24 \mathrm{~h}$ as detected by real-time PCR.

A

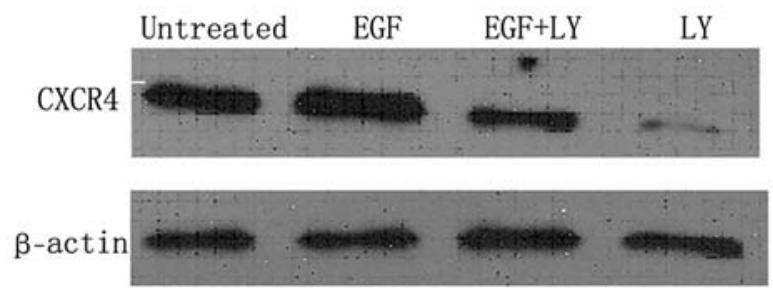

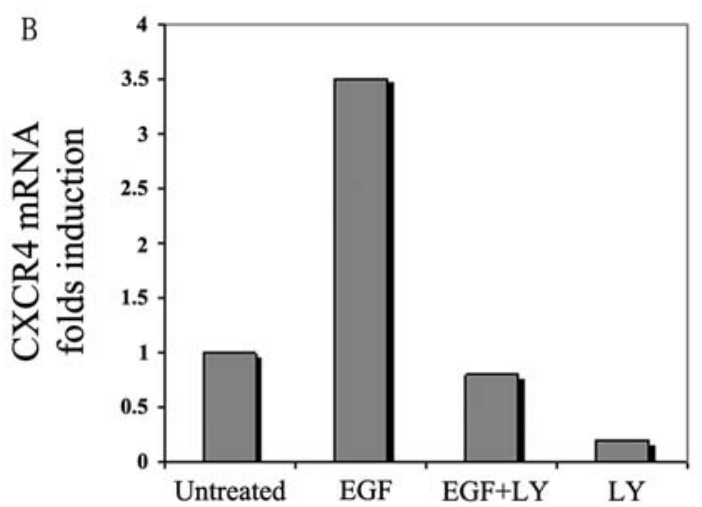

Figure 5. PI-3 kinase inhibitor (LY294002) blocks EGF-induced upregulation of CXCR4. A) Western blot analysis of CXCR4 protein. (B) Real-time PCR analysis of CXCR4 mRNA. Untreated, without addition of LY294002 and EGF; EGF, addition of EGF only; EGF/LY, addition of LY294002 and EGF; LY, addition of LY294002 only.

EGFR (CXCR4 $\left.{ }^{+} / \mathrm{EGFR}^{-}\right)$, group III with negative CXCR4 and positive EGFR (CXCR4/EGFR ${ }^{+}$) and group IV with negative CXCR4 and negative EGFR (CXCR4/EGFR').

The median survival times among these groups were: group I $\left(\mathrm{CXCR}^{+} / \mathrm{EGFR}^{+}\right), 11.6$ months; group II (CXCR4 ${ }^{+} /$ EGFR $\left.{ }^{-}\right), 14.86$ months; group III (CXCR4-/EGFR $\left.{ }^{+}\right), 19.8$ months and group IV (CXCR4/EGFR'), 24.8 months. The estimated risk of death for these groups adjusted for patient age, tumor stage and type of treatments that patients received is shown in Table II.

The incidence of death was not different in patients with $\mathrm{EGFR}^{+}$when compared with EGFR- $(\mathrm{HR}=1.33, \mathrm{P}=0.248)$, but the addition of CXCR4 expression resulted in a statistically significant increase in the incidence of death in group I patients $\left(\mathrm{CXCR} 4^{+} / \mathrm{EGFR}^{+}\right)$when compared with the other groups $(\mathrm{HR}=2.415, \mathrm{P}=0.01)$. Furthermore, when we compared group I patients $\left(\mathrm{CXCR} 4^{+} / \mathrm{EGFR}^{+}\right)$with group IV patients (CXCR4 ${ }^{-/ E G F R}{ }^{-}$), the incidence of cancer-related death was much higher (HR=2.741, $\mathrm{P}=0.006$ ) (Fig. 3).

When we compared group II (CXCR4 $4^{+} / \mathrm{EGFR}^{-}$) or III $\left(\mathrm{CXCR} 4 / \mathrm{EGFR}^{+}\right)$with the other groups, no significant differ- ence was found concerning the risk of death ( $\mathrm{HR}=1.197$, $\mathrm{P}=0.533$ and $\mathrm{HR}=0.953, \mathrm{P}=0.199$, respectively). The risk of death was reduced in group IV patients (CXCR4/EGFR-) when compared with the other groups, yet this reduction was not statistically significant $(\mathrm{HR}=0.688, \mathrm{P}=0.188)$.

Expression of CXCR4 and EGFR in a human NSCLC cell line. Total RNA was isolated from the A549 cell line, and reverse transcriptase PCR was performed to evaluate the expression of CXCR4 and EGFR. CXCR4 and EGFR mRNAs were highly expressed in the A549 cell line (Fig. 4A).

Expression of CXCR4 in A549 cells after stimulation by EGF. A549 cells (cultured in RPMI starved media for $24 \mathrm{~h}$ ) were stimulated with different concentrations of EGF for an additional $24 \mathrm{~h}$, and CXCR4 expression levels were then evaluated by real-time PCR. When compared to the control, the expression of CXCR4 mRNA was found to be concentration-dependent (Fig. 4B). To confirm that CXCR4 upregulation was secondary to EGF, A549 cells were treated with AG1478 (a specific inhibitor of EGFR phosphorylation) $(10 \mu \mathrm{M})$ for $24 \mathrm{~h}$, and CXCR4 expression was 
re-evaluated by real-time PCR and western blotting (Fig. 4C and D). Our results demonstrate that CXCR4 mRNA and protein levels were reduced after treatment with AG1478.

Role of the PI-3 kinase pathway in EGF-induced upregulation of CXCR4 expression in A549 cells. EGF was previously shown to enhance the expression of CXCR4 through the PI-3 kinase pathway, therefore we hypothesized that EGF induces upregulation of CXCR4 through the PI-3 kinase pathway in NSCLC. Hence, to prove this hypothesis, serum-starved A549 cells were treated with EGF in the presence or absence of LY294002 (a PI-3 kinase pathway inhibitor). Cells were cultured in serum-starved media for $24 \mathrm{~h}$, pretreated or not for $2 \mathrm{~h}$ with LY294002, then stimulated with EGF $(40 \mathrm{ng} / \mathrm{ml})$ for $24 \mathrm{~h}$. CXCR4 expression was assessed by real-time PCR and western blotting (Fig. 5). Our results showed that the EGF-enhanced upregulation of CXCR4 was blocked by inhibition of the PI-3 kinase pathway.

\section{Discussion}

Research has demonstrated that cross-talk between EGFR and CXCR4 is important in the proliferation and metastasis of ovarian and breast cancers $(7,27,28)$. Therefore, in this study, we investigated the correlation between EGFR and CXCR4 in a human NSCLC cell line and tumor specimens, and also analyzed the correlation between co-expression of both molecules (EGFR and CXCR4), as compared with the high expression of a single molecule alone using immunohistochemical staining.

Previously, several studies have shown an association between the expression of the EGFR protein and patient survival in NSCLC (34). However, other studies have either demonstrated no significant association $(35,36)$, or an inverse relationship between the expression of EGFR protein and survival (5). A meta-analysis reported by Meert et al showed that an average of $51 \%$ of NSCLC cases exhibited overexpression of the EGFR protein in 14 studies based on immunohistochemical analysis (37). Twelve of the 16 studies (approximately three-fourths) did not find EGFR expression to be a prognostic indicator. Similarly, Nicholson et al (38) carried out another review and included studies that were published from 1985 to 2000 . They demonstrated that $20 \%$ of the studies found an association between increased EGFR expression and a lower recurrence-free survival in NSCLC, whereas, only $10 \%$ of the studies showed an association between the overexpression of the EGFR protein and overall survival.

In the present study, patients with EGFR ${ }^{+}$had a higher estimated risk of death than those patients with EGFR $(\mathrm{HR}=1.33)$, but this trend did not reach statistical significance $(\mathrm{P}=0.248)$. These findings remain controversial as many studies did not adjust for significant prognostic factors such as tumor stage, patient age at diagnosis and type of treatment. In contrast, our study did address the relationship between EGFR expression and clinical variables. Furthermore, many studies suggest that the improvement in survival among patients with high expression of EGFR is associated with enhanced responsiveness to anti-EGFR-targeting therapies (5) In contrast, all of the patients that were included in our study had not received any type of target therapy.
Previous studies have attempted to determine the clinical significance of CXCR4 expression in NSCLC. Spano et al, studying only stage I lesions, found a significant association between nuclear CXCR4 staining and prolonged survival of patients with NSCLC (32). Su et al showed a significant correlation between high cytoplasmic CXCR4 staining intensity and metastasis (18). Recently, Wagner et al demonstrated an independent association between CXCR4 expression and survival depending on its subcellular location; nuclear staining was correlated with good prognosis, whereas cytomembranous staining was correlated with poor prognosis (19). Our results are consistent with previous studies. We found that patients with cytoplasmic overexpression of CXCR4 had a significant association with metastasis and had a significantly shorter overall survival and a high incidence of cancer-related death. However, we found nuclear staining in only $15.2 \%$ of patients with early-stage disease, and these patients had a comparatively long overall survival. This is quite reasonable since only $31 \%$ of the patients included in our study had stage I and II disease which is consistent with previous studies that demonstrated that strong nuclear CXCR4 staining is associated with a good prognosis in patients with early-stage NSCLC (32).

Furthermore, our study analyzed the clinicopathological and prognostic significance of co-expression of CXCR4 and EGFR in NSCLC. A positive correlation was found between co-expression of the two molecules and distant metastasis and advanced stage disease $(\mathrm{P}=0.005)$. Moreover, patients with co-expression of EGFR and CXCR4 significantly had shorter OS (11.6 months) when compared to the other groups: $\mathrm{CXCR}^{+} / \mathrm{EGFR}^{-}, \mathrm{CXCR}^{2} / \mathrm{EGFR}^{+}$and $\mathrm{CXCR}^{-} / \mathrm{EGFR}^{-}$. Additionally, patients with $\mathrm{CXCR} 4^{+} / \mathrm{EGFR}^{+}$had a higher risk of cancer-related death $(\mathrm{HR}=2.415)$, whereas the risk of death for patients with only EGFR ${ }^{+}$or CXCR $4^{+}$was lower $(\mathrm{HR}=1.33$ and 2.172 , respectively). These results suggest that concomitant overexpression of EGFR and CXCR4 is associated with poorer prognosis as compared with that of high expression of a single molecule.

To our knowledge, this is the first study to investigate the dual CXCR4/EGFR tumor status and determine its prognostic impact in NSCLC. These results suggest a possible important relationship between CXCR4 and EGFR intracellular pathways that may stimulate different cell proliferation- and metastasis-related pathways in NSCLC. Based on EGFR and CXCR4 expression in our study, patients with CXCR4/EGFR had the most favorable prognosis followed by CXCR4 $/ \mathrm{EGFR}^{+}$ and $\mathrm{CXCR} 4^{+} / \mathrm{EGFR}^{-}$. Patients with $\mathrm{CXCR} 4^{+} / \mathrm{EGFR}^{+}$were associated with a worse prognosis.

Noteworthy, similar results were found in previous studies regarding other tumor types. High expression of EGFR and CXCR4 was found to be associated with increased risk of death and recurrence in inflammatory breast cancer (30). Another study demonstrated that combined high expression of cytoplasmic CXCR4 and VEGFR was significantly associated with lymphatic and distant metastasis in colorectal cancer (31). This suggests the existence of a shared mechanism of CXCR4 and EGFR interaction in common epithelial malignancies. Similarly, CXCR4 was shown to be frequently expressed with EGFR in synovial sarcoma (39).

An interaction between EGFR and CXCR4 has been noted in many types of cancer. Regarding NSCLC, Phillips et al 
emphasized that activation of EGFR by EGF increased CXCR4 expression in normoxia and hypoxia in NSCLC (40) In the present study, our results are consistent with this finding. We demonstrated that EGF upregulated CXCR4 expression through the PI-3 kinase pathway. Furthermore, we showed that CXCR4 mRNA upregulation increased in a concentration-dependent manner. In addition, CXCR4 expression was reduced when A549 cells were treated with an EGFR phosphorylation inhibitor (AG1478). These results further confirm the relationship between EGFR and CXCR4 and explain the reason as to why higher tumor grades and a shorter overall survival rate are noted in patients with co-expression of CXCR4 and EGFR.

The mechanisms of EGF-induced CXCR4 upregulation are not yet well understood, and may be explained by the fact that mechanisms involved in CXCR4 ubiquitination and sorting may share some common features with EGFR endocytosis and degradation processes, although they are structurally unrelated membrane receptors. Internalization of CXCR4 through early endosomes, and then sorting into late endosomes and lysosomes is one mechanism of CXCR4 degradation. Therefore, we suggest that EGFR may inhibit the process of CXCR4 ubiquitination and subsequently abrogate sorting and prevent its degradation (41). Taken together, regulation of CXCR4 expression can be accomplished through different mechanisms. When and where each mechanism should be used to regulate CXCR4 expression warrants further exploration.

In conclusion, according to our data, NSCLC tumor cells with concomitant expression of both CXCR4 and EGFR may represent a subpopulation that is able to achieve a more aggressive clinical progression. Furthermore, CXCR4 and EGFR could be used as new biomarkers for indicating poor prognosis in NSCLC. However, due to the limited percentage of patients with co-expression of both CXCR4 and EGFR and the small sample size of our study, these results must be confirmed in large prospective studies with cautious interpretation. It will also be worthwhile to evaluate the efficacy of different treatments targeting both CXCR4 and EGFR in comparison with tyrosine kinase inhibitor treatment in patients with concomitant overexpression of CXCR4 and EGFR.

\section{Acknowledgements}

This study was supported from the Hubei Provincial Natural Science Foundation of China (no. 2008CDA065).

\section{References}

1. Chen G, Wang Z, Liu XY and Liu FY: High-level CXCR4 expression correlates with brain-specific metastasis of non-small cell lung cancer. World J Surg 35: 56-61, 2011.

2. Dong J, Dai J, Shu Y, et al: Polymorphisms in EGFR and VEGF contribute to non-small-cell lung cancer survival in a Chinese population. Carcinogenesis 31: 1080-1086, 2010.

3. Yang L, Li L, Chen Y and Parkin DM: Mortality time trends and the incidence and mortality estimation and projection for lung cancer in China. Zhongguo Fei Ai Za Zhi 8: 274-278, 2005 (In Chinese)

4. Yang L, Parkin DM, Ferlay J, Li L and Chen Y: Estimates of cancer incidence in China for 2000 and projections for 2005. Cancer Epidemiol Biomarkers Prev 14: 243-250, 2005.

5. Van Dyke AL, Cote ML, Prysak GM, et al: COX-2/EGFR expression and survival among women with adenocarcinoma of lung. Carcinogenesis 29: 1781-1787, 2008.
6. Sung B, Jhurani S, Ahn KS, et al: Zerumbone down-regulates chemokine receptor CXCR4 expression leading to inhibition of CXCL12-induced invasion of breast and pancreatic tumor cells. Cancer Res 68: 8938-8944, 2008.

7. Rahimi M, George J and Tang C: EGFR variant-mediated invasion by enhanced CXCR4 expression through transcriptional and posttranslational mechanisms. Int J Cancer 126: 1850-1860, 2010.

8. Tang $\mathrm{CH}$, Tan TW, Fu WM and Yang RS: Involvement of matrix metalloproteinase-9 in stromal cell-derived factor-1/CXCR4 pathway of lung cancer metastasis. Carcinogenesis 29: 35-43, 2008.

9. Kryczek I, Wei S, Keller E, Liu R and Zou W: Stromal-derived factor (SDF-1/CXCL2) and human tumor pathogenesis. Am J Physiol Cell Physiol 292: C987-C995, 2007.

10. Imai H, Sunaga N, Shimizu Y, et al: Clinicopathological and therapeutic significance of CXCL12 expression in lung cancer. Int J Immunopathol Pharmacol 23: 153-164, 2010.

11. Andre F, Xia W, Conforti R, et al: CXCR4 expression in early breast cancer and risk of distant recurrence. Oncologist 14: 1182-1188, 2009.

12. Chinni RS, Yamamoto H, Dong Z, et al: CXCL12/CXCR4 transactivates HER2 in lipid rafts of prostate cancer cells and promotes growth of metastatic deposit in bone. Mol Cancer Res 6: 446-457, 2008.

13. Wong D and Korz W: Translating an antagonist of chemokine receptor CXCR4: from bench to bedside. Clin Cancer Res 14: 7975-7980, 2008.

14. Oonakahara K, Matsuyama W, Higashimoto I, et al: Stromalderived factor-1alpha/CXCL12-CXCR 4 axis is involved in the dissemination of NSCLC cells into pleural space. Am J Respir Cell Mol Biol 30: 671-677, 2004.

15. Kijima T, Maulik G, Ma PC, et al: Regulation of cellular proliferation, cytoskeletal function, and signal transduction through CXCR4 and c-Kit in small cell lung cancer cells. Cancer Res 62: 6304-6311, 2002.

16. Phillips RJ, Burdick MD, Lutz M, et al: The stromal derived factor-1/CXCL12-CXC chemokine receptor 4 biological axis in non-small cell lung cancer metastases. Am J Respir Crit Care Med 167: 1676-1686, 2003.

17. Martínez-García E, Irigoyen M, González-Moreno O, et al: Repetitive nicotine exposure leads to a more malignant and metastasis-prone phenotype of SCLC: a molecular insight into the importance of quitting smoking during treatment. Toxicol Sci 116: 467-476, 2010.

18. Su L, Zhong J, Xu H, et al: Differential expression of CXCR4 is associated with the metastatic potential of human non-small cell lung cancer cells. Clin Cancer Res 11: 8273-8280, 2005.

19. Wagner PL, Hyjek E, Vazquez MF, et al: CXCL12 and CXCR4 in adenocarcinoma of the lung: association with metastasis and survival. J Thorac Cardiovasc Surg 137: 615-621, 2009.

20. Cappuzzo F, Hirsch FR, Rossi E, et al: Epidermal growth factor receptor gene and protein and gefitinib sensitivity in non-smallcell lung cancer. J Natl Cancer Inst 97: 643-655, 2005.

21. Morita S, Okamoto I, Kobayashi K, et al: Combined survival analysis of prospective clinical trials of gefitinib for non-small cell lung cancer with EGFR mutation. Clin Cancer Res 15: 4493-4498, 2009.

22. Liang Z, Zhang J, Zeng X, et al: Relationship between EGFR expression, copy number and mutation in lung adenocarcinomas. BMC Cancer 10: 376, 2010.

23. Suzuki S, Dobashi Y, Yamane T, et al: Protein overexpression and gene amplification of epidermal growth factor receptor in nonsmall cell lung carcinomas. An immunohistochemical and fluorescence in situ hybridization study. Cancer 103: 1265-1273, 2005.

24. Fischer OM, Hart S, Gschwind A and Ullrich A: EGFR signal transactivation in cancer cells. Biochem Soc Trans 31: 1203-1208, 2003.

25. Zaman SN, Resek ME and Robbins SM: Dual acylation and lipid raft association of Src-family protein tyrosine kinases are required for SDF-1/CXCL12-mediated chemotaxis in the Jurkat human T cell lymphoma cell line. J Leukoc Biol 84: 1082-1091, 2008.

26. Peipp M, Schneider-Merck T, Dechant M, et al: Tumor cell killing mechanisms of epidermal growth factor receptor (EGFR) antibodies are not affected by lung cancer-associated EGFR kinase mutations. J Immunol 180: 4338-4345, 2008.

27. Porcile C, Bajetto A, Barbieri F, et al: Stromal cell-derived factor-1alpha (SDF-1alpha/CXCL12) stimulates ovarian cancer cell growth through the EGF receptor transactivation. Exp Cell Res 308: 241-253, 2005. 
28. Guo Z, Cai S, Fang R, et al: The synergistic effects of CXCR4 and EGFR on promoting EGF-mediated metastasis in ovarian cancer cells. Colloids Surf B Biointerfaces 60: 1-6, 2007.

29. Cabioglu N, Summy J, Miller C, et al: CXCL-12/stromal cellderived factor-1alpha transactivates HER2-neu in breast cancer cells by a novel pathway involving Src kinase activation. Cancer Res 65: 6493-6497, 2005.

30. Cabioglu N, Gong Y, Islam R, et al: Expression of growth factor and chemokine receptors: new insight in the biology of inflammatory breast cancer. Ann Oncol 18: 1021-1029, 2007.

31. Wu Y G, Jin M, Xu H, et al: Clinicopathologic significance of HIF-1 $\alpha$, CXCR4, and VEGF expression in colon cancer. Clin Dev Immunol 7: 537531, 2010

32. Spano JP, Andre F, Morat L, et al: Chemokine receptor CXCR4 and early-stage non-small cell lung cancer: pattern of expression and correlation with outcome. Ann Oncol 15: 613-617, 2004.

33. Ludovini V, Bellezza G, Pistola L, et al: High coexpression of both insulin-like growth factor receptor-1 (IGFR-1) and epidermal growth factor receptor (EGFR) is associated with shorter disease-free survival in resected non-small-cell lung cancer patients. Ann Oncol 20: 842-849, 2009.

34. Niemiec J, Kolodziejski L and Dyczek S: EGFR LI and Ki-67 LI are independent prognostic parameters influencing survivals of surgically treated squamous cell lung cancer patients. Neoplasma 52: 231-237, 2005.
35. Brabender J, Danenberg KD, Metzger R, et al: Epidermal growth factor receptor and HER2-neu mRNA expression in non-small cell lung cancer is correlated with survival. Clin Cancer Res 7: 1850-1855, 2001.

36. Kanematsu T, Yano S, Uehara H, et al: Phosphorylation, but not overexpression, of epidermal growth factor receptor is associated with poor prognosis of non-small cell lung cancer patients. Oncol Res 13: 289-298, 2003.

37. Meert AP, Martin B, Delmotte P, et al: The role of EGF-R expression on patient survival in lung cancer: a systematic review with meta-analysis. Eur Respir J 20: 975-981, 2002.

38. Nicholson RI, Gee JM and Harper ME: EGFR and cancer prognosis. Eur J Cancer 37 (Suppl 4): S9-S15, 2001

39. Grisanti S, Rossi L, Ardighieri C, et al: CXCR4, EGFR and HER2 expression in patients with high-risk synovial sarcoma (SS): a clinicopathologic study. J Clin Oncol 24: 9580, 2006.

40. Phillips RJ, Mestas J, Gharaee-Kermani M, et al: Epidermal grow th factor and hypoxia-induced expression of CXC chemokine receptor 4 on non-small cell lung cancer cells is regulated by the phosphatidylinositol 3-kinase/PTEN/AKT/mammalian target of rapamycin signaling pathway and activation of hypoxia inducible factor-1alpha. J Biol Chem 280: 22473-22481, 2005.

41. Li YM, Pan Y, Wei Y, et al: Upregulation of CXCR4 is essential for HER2-mediated tumor metastasis. Cancer Cell 6: 459-469, 2004. 